\title{
Homicidios intencionais de mulheres com notificação prévia de violência
}

Intentional homicides of women with prior notification of violence Homicidios intencionales de mujeres con notificación previa de violencia

Sheyla Carvalho de Barros ${ }^{1}$ @ h htps://orcid.org/0000-0001-5399-0296

Dayane da Rocha Pimentel2 Đi https://orid.org/0000-0002-9863-134X

Conceição Maria de Oliveira', ${ }^{3,4}$ https:///orcid.org/0000-0002-2220-5782

Cristine Vieira do Bonfim ${ }^{1,5}$ ic https://orcid.org/0000-0002-4495-9673

Como citar:

Barros SC, Pimentel DR, Oliveira CM, Bonfim

CV. Homicídios intencionais de mulheres com notificação prévia de violência. Acta Paul Enferm. 2021;34:AAPE00715.

DOI

http://dx.doi.org/10.37689/actaape/2021A000715

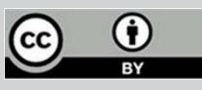

Descritores

Homicídio; Violência contra a mulher; Sistemas de Informação em Saúde; Estatísticas vitais; Registros

de mortalidade

Keywords

Homicide; Violence against women; Helath information systems; Vital statistics; Mortality

registries

Descriptores

Homicidio; Violencia contra la mujer; Sistemas de información en salud; Estadísticas vitales; Registros

de mortalidad

Submetido

4 de Abril de 2020

Aceito

2 de Dezembro de 2020

Autor correspondente

Sheyla Carvalho de Barros

E-mail: sheyla.barross@gmail.com

\section{Resumo}

Objetivo: Descrever o perfil das mulheres vítimas de homicídios intencionais e com notificação prévia de violência.

Métodos: Trata-se de um estudo transversal, realizado no estado de Pernambuco, no período de 2012 a 2016. Foi empregado o relacionamento probabilístico entre todas as notificações de violência contra mulher, registradas no Sistema de Informação de Agravos de Notificação, e os homicídios de mulheres, registrados no Sistema de Informações sobre Mortalidade.

Resultados: Identificou-se 121 homicídios que tinham notificações prévias de violência. As mulheres eram solteiras (88,9\%), negras (91,7\%) e com menos de sete anos de estudo (80,9\%). A agressão física foi o tipo de violência mais notificado $(65,8 \%)$, ocorrida na residência $(66,7 \%)$ e cometida por parceiro/ex-parceiro íntimo (51,9\%). 0 disparo de arma de fogo foi o principal meio utilizado $(44,6 \%)$ e 0 óbito ocorreu em estabelecimento de saúde (41,3\%). Mulheres com notificação prévia de violência tiveram risco 65,9 vezes maior de homicídio, quando comparadas com a população geral de mulheres.

Conclusão: Descrever o perfil das mulheres vítimas de homicídios, com notificação de violência prévia, pode contribuir para a formulação de políticas públicas de proteção e prevenção da violência contra mulher.

\section{Abstract}

Objective: To describe the profile of women victims of intentional homicides and with prior notification of violence.

Methods: This is a cross-sectional study carried out in the state of Pernambuco from 2012 to 2016. Probabilistic relationship was used between all notifications of violence against women registered in the Information System for Notifiable Diseases, and the homicides of women, registered in the Mortality Information System.

Results: 121 homicides were identified with previous reports of violence. Women were single (88.9\%), black $(91.7 \%)$ and had less than seven years of study (80.9\%). Physical aggression was the most reported type of violence $(65.8 \%)$, occurring at home $(66.7 \%)$ and committed by a partner/ex-intimate partner $(51.9 \%)$. Firearm firing was the main method used $(44.6 \%)$ and death occurred in a health facility (41.3\%). Women with prior notification of violence had a 65.9 times higher risk of homicide when compared to the general population of women.

Conclusion: Describing the profile of women victims of homicides, with prior notification of violence, can contribute for formulating public policies for the protection and prevention of violence against women. 


\section{Resumen}

Objetivo: Describir el perfil de mujeres víctimas de homicidios intencionales con notificación previa de violencia.

Métodos: Se trata de un estudio transversal, realizado en el estado de Pernambuco, en el período de 2012 a 2016. Se empleó la relación probabilística entre todas las notificaciones de violencia contra la mujer registradas en el Sistema de Información de Agravios de Notificación y los homicidios de mujeres registrados en el Sistema de Información sobre Mortalidad.

Resultados: Se identificaron 121 homicidios que tenían notificaciones previas de violencia. Las mujeres eran solteras (88,9\%), negras (91,7 \%) y con menos de siete años de estudios (80,9\%). La agresión física fue el tipo de violencia más notificado (65,8 \%), ocurrida en la residencia (66,7 \%) y cometida por la pareja/expareja íntima (51,9\%). El disparo de arma de fuego fue el principal medio utilizado (44,6 \%) y la muerte ocurrió en un establecimiento de salud (41,3\%). Mujeres con notificación previa de violencia tuvieron un riesgo de homicidio 65,9 veces mayor, en comparación con la población general de mujeres.

Conclusión: Describir el perfil de mujeres víctimas de homicidios con notificación de violencia previa puede contribuir a la formulación de políticas públicas de protección y prevención de la violencia contra la mujer.

\section{Introdução}

A mortalidade é condicionada por fatores biológicos, políticos e sociais, bem como por comportamentos e atitudes culturalmente definidos. ${ }^{(1)} \mathrm{A}$ violência contra as mulheres é a mais difundida, porém a menos reconhecida violação dos direitos humanos no mundo. É um profundo problema de saúde e o homicídio, ou feminicídio, muitas vezes, é o ponto final trágico dessa violência. ${ }^{(1,2)}$

A violência por parceiros íntimos afeta entre $15 \%$ e $71 \%$ das mulheres ao longo de suas vidas. As características incluem abuso físico e sexual, bem como abuso psicológico e coerçáo reprodutiva ou perseguição. ${ }^{(3)}$ Estima-se que de $40 \%$ a $45 \%$ das vítimas sejam mortas por um parceiro íntimo. ${ }^{(2)}$

Os serviços de saúde têm papel importante para identificação e resposta às diversas situações de violência, pois, muitas vezes, são os primeiros locais onde as vítimas buscam atendimento. ${ }^{(4,5)}$ No Brasil, no período de 2009 a 2017, foram notificadas, nos serviços de saúde, 1.091.264 mulheres vítimas dos diversos tipos de violência. ${ }^{(6)}$ Em relação aos homicídios, no período de 2007 a 2017, houve um crescimento no número, passando de 3.778 para 4.936 (30,7\%). Nesse mesmo período, a taxa nacional de homicídios passou de 3,9 para 4,7 mulheres assassinadas por 100 mil mulheres, representando aumento de 20,7\%. Registre-se que também ocorreu aumento na taxa de mortalidade em 17 unidades da federação. ${ }^{(7)}$

A vinculação de registros é o processo usado para reunir informaçóes, de diferentes fontes, sobre o mesmo indivíduo ou grupo de indivíduos. ${ }^{(8)}$ Devido à crescente disponibilidade de bancos de dados de saúde baseados na população, a articulação tornou-se uma ferramenta de pesquisa eficiente e importante. ${ }^{(9)}$
Há duas abordagens principais para a vinculação de bases: determinística e probabilística. A vinculação determinística é realizada usando um identificador único, presente nos arquivos que se deseja vincular. ${ }^{(10)} \mathrm{A}$ probabilística é útil quando não existe o identificador único. Esse tipo de vinculação combina a evidência por meio de um número de identificadores, representando a probabilidade de dois registros pertencerem à mesma pessoa. ${ }^{(8,9)}$

Uma área de pesquisa para a qual a vinculação de dados é particularmente importante é a da violência contra a mulher. Nos Estados Unidos, o linkage foi usada para analisar a relação entre mortes violentas e admissões anteriores, nos serviços de emergência, por lesóes decorrentes de causas externas. ${ }^{(11)}$ Também foi importante para localizar os óbitos maternos que estavam associados às mortes violentas. ${ }^{(12)}$ No Brasil, aplicou-se o linkage das bases de dados do Sistema de Informaçóes sobre Mortalidade e do Sistema de Informaçáo sobre Nascidos Vivos, para analisar violência sexual e suas posteriores repercussóes na gravidez e parto. ${ }^{(13)}$

Conhecer eventos anteriores aos homicídios intencionais de mulheres possibilita melhor compreensão das circunstâncias e causas da morte. As informaçōes obtidas com a vinculação dos bancos de dados do Sistema de Informaçóes sobre Mortalidade e do Sistema de Informação de Agravos de Notificaçáo podem ser usadas para o planejamento de açóes da rede intersetorial, que atua no enfretamento da violência contra mulheres. Nessa perspectiva, o problema de pesquisa é: qual o perfil das mulheres vítimas de homicídios intencionais e que tinham notificação prévia de violência? Este estudo tem como objetivo descrever o perfil das mulheres vítimas de homicídios intencionais e com notificação prévia de violência. 


\section{Métodos}

Trata-se de um estudo transversal, realizado no estado de Pernambuco, que possui 184 municípios e um distrito - o arquipélago de Fernando de Noronha. De acordo com a estimativa populacional do Instituto Brasileiro de Geografia e Estatística, a população total é de 9.410 .336 habitantes. As mulheres são maioria, 4.873.140, equivalente a 52\% do total da populaçáo. ${ }^{(14)}$

A população do estudo foi constituída por mulheres (incluindo crianças e adolescentes do sexo feminino a partir dos 10 anos de idade), vítimas de homicídios intencionais, entre 2012 e 2016, residentes em Pernambuco. A escolha do período de análise, iniciando no ano de 2012, deveu-se ao fato de que, em 2011, ocorreu uma mudança no conteúdo da declaração de óbito, com maior detalhamento das informaçóes coletadas. $\mathrm{O}$ ano de 2016 correspondeu ao último ano com dados finalizados (não provisórios), disponíveis no Sistema de Informaçóes sobre Mortalidade, no momento de realização do estudo.

Foram incluídos os óbitos classificados no Sistema de Informaçóes sobre Mortalidade, dentro do Capítulo XX (Causas externas de morbidade e de mortalidade) da Classificação Estatística Internacional de Doenças e Problemas Relacionados à Saúde (CID-10), com os códigos X85 a Y09 (agressões), e que tinham notificaçôes de algum tipo de violência no Sistema de Informação de Agravos de Notificação, entre os anos 2011 e 2016. Utilizou-se as projeçôes populacionais para o cálculo da taxa de mortalidade por homicídios, no período de 2012 a $2016 .^{(15)}$

Para o relacionamento das bases de dados, considerando que os sistemas utilizados não possuem um campo identificador único, foi aplicado o método probabilístico. No banco do Sistema de Informaçóes sobre Mortalidade, além dos homicídios, optou-se por também incluir, no relacionamento, os óbitos classificados no Capítulo XX com os códigos Y10 a Y34 (eventos/fatos cuja intenção é indeterminada), visando refinar os dados da pesquisa.

Foi adotado como critério de exclusão: (a) casos em que as variáveis "nome" e "nome da mãe" não estavam preenchidos, pois impossibilitava a identifi- cação dos pares; (b) casos notificados no Sistema de Informação de Agravos de Notificação envolvendo violência autoprovocada; (c) casos onde a agressáo notificada refere-se ao homicídio (notificaçáo do homicídio).

Foram consideradas como variáveis de pareamento "nome", "nome da mãe" e "data de nascimento". O programa RecLink III foi usado para a aplicação do linkage, executado em etapas que se iniciaram pela limpeza das bases de dados, seguida do cálculo de escores, que é o procedimento para a definição de limiares para a classificação dos pares de registros, relacionados em pares verdadeiros, não pares e pares duvidosos. ${ }^{(16)}$

$\mathrm{Na}$ etapa seguinte, foram padronizadas as variáveis: nome, idade, data de nascimento, endereço, bairro e município de residência, seguida da subdivisão dos campos e criação dos campos fonéticos. A etapa subsequente foi a blocagem de registros, que subdivide os arquivos de acordo com a chave de indexaçáo, formadas a partir das variáveis de pareamento. As comparaçóes de registros são restritas à concordância do valor das chaves. Os pares verdadeiros e os duvidosos foram revisados manualmente. Após a vinculação, foi possível identificar, entre os homicídios, os que possuíam registros prévios de violência.

Foram calculadas taxas de mortalidade por agressão, em mulheres com registros de violência prévia e na população em geral. As seguintes fórmulas foram utilizadas: taxa de mortalidade por agressão na população de mulheres em geral $=$ média de óbitos de mulheres por agressão/população média de mulheres x 100.000 mulheres; taxa de mortalidade por agressão em mulheres com registros prévios de violência = óbitos de mulheres por agressão com notificação de violência prévia/notificações de violência de mulheres x 100.000 mulheres; razão das taxas de mortalidade por agressão $=$ taxa de mortalidade por homicídios em mulheres com registros prévios de violência/taxa de mortalidade por agressão na populaçáo de mulheres em geral. ${ }^{(16)}$

Este estudo foi aprovado pelo Comitê de Ética em Pesquisa envolvendo seres humanos, da Universidade Federal de Pernambuco (CAAE 91858618.9.0000.5208), parecer no 2.986 .711 . 


\section{Resultados}

Foram registradas 32.308 notificações de violência no Sistema de Informação de Agravos de Notificação, 1.162 homicídios e 901 óbitos por causas externas de intenção indeterminada no Sistema de Informações sobre Mortalidade. Após a vinculação, foram relacionados 200 pares, dos quais 79 foram excluídos por estarem identificados, no Sistema de Informação de Agravos de Notificação, como violência autoprovocada ou por serem a notificação do próprio homicídio. Verificou-se 121 óbitos que possuíam notificaçóes prévias de violência, sendo 101 pares de homicídios e 20 pares com óbitos de causas externas de intençáo indeterminada (Figura 1).

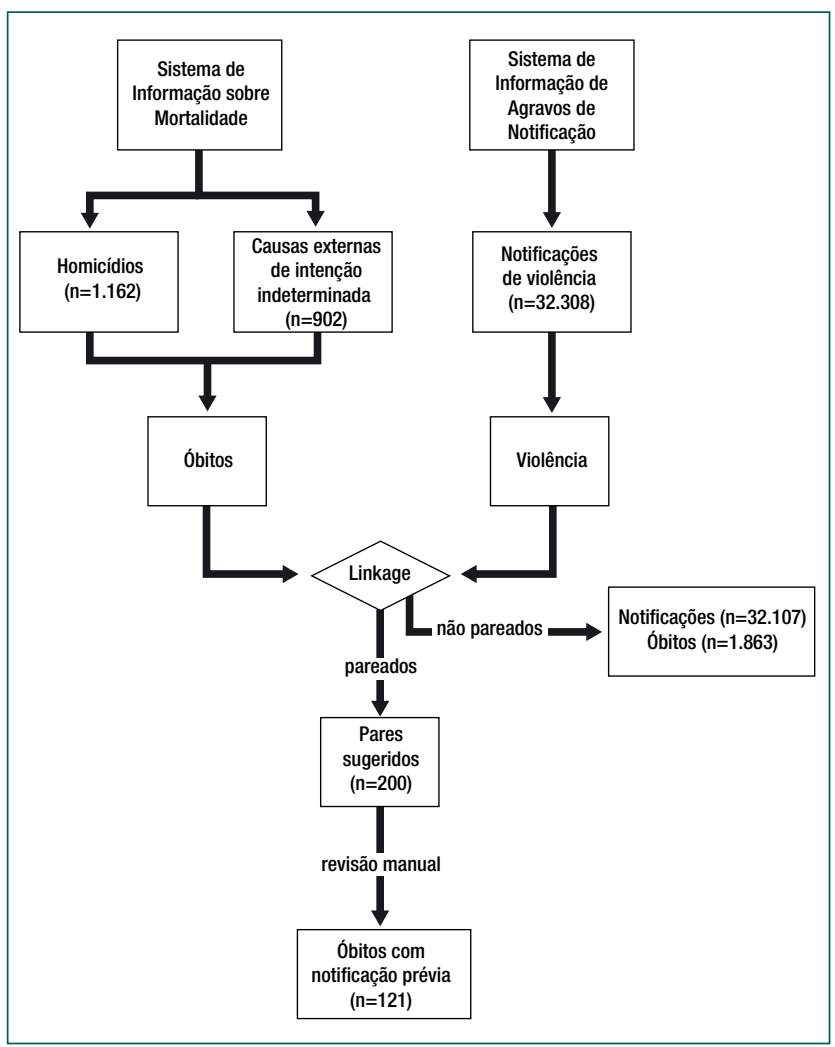

Figura 1. Fluxograma do linkage entre 0 Sistema de Informações sobre Mortalidade e o Sistema de Informação de Agravos de Notificação

Os homicídios com notificação prévia de violência representaram $8,7 \%$ dos casos. O tempo entre a notificação e o homicídio foi, em média, de 17 meses (desvio padrão de 19,4), sendo de três dias o menor intervalo encontrado e de cinco anos, o maior.

Para a população geral de mulheres, a taxa de homicídios foi de 5,7 por 100.000 mulheres. Para a as vítimas que tinham notificação prévia de violência, a taxa foi de 374,5 por 100.000 mulheres. Por meio da razão das taxas, observou-se que mulheres que tinham notificação prévia de violência apresentaram risco 65,9 vezes maior de homicídio do que a população de mulheres em geral (Tabela 1).

Tabela 1. Taxa de homicídios na população geral de mulheres, taxa de homicídios em mulheres com notificação prévia de violência e razão das taxas

\begin{tabular}{lc}
\hline População & Taxa \\
\hline População geral de mulheres & 5,7 \\
Mulheres com notificação prévia de violência & 374,5 \\
Razão das taxas & 65,9 \\
\hline
\end{tabular}

A tabela 2 apresenta as características dos óbitos com notificação prévia de violência. A maior parte das vítimas era maior de 20 anos $(82,6 \%)$, solteira $(88,9 \%)$, negra $(91,7 \%)$ e com menos de sete anos de estudo $(80,9 \%)$. Com relação a notificação prévia, observou-se que a maior frequência era violência do tipo física $(65,8 \%)$, por meio de força corporal $(42,2 \%)$, cometida por parceiro/ex-parceiro íntimo $(51,9 \%)$ e sem registro de reincidência $(63,6 \%)$. Os óbitos ocorreram no hospital ou outros serviços de saúde $(41,3 \%)$. A principal causa de óbito foi por meio de disparo de arma de fogo $(44,6 \%)$, seguida de objeto cortante ou penetrante $(25,6 \%)$.

\section{Discussão}

Os resultados deste estudo identificaram mulheres negras, solteiras, que sofreram agressóes físicas perpetradas por um parceiro íntimo, atual ou antigo, com o uso de arma de fogo. O risco de homicídio para as mulheres que tinham notificação prévia de violência foi superior ao das mulheres da população geral.

Mulheres que tinham notificação prévia de violência apresentaram risco 65,9 vezes maior de homicídio do que a população de mulheres em geral. Pesquisa realizada no Brasil, revelou que 
Tabela 2. Características dos homicídios com registros prévios de violência após linkage

\begin{tabular}{|c|c|}
\hline Variáveis & $\mathrm{n}(\%)$ \\
\hline \multicolumn{2}{|l|}{ Faixa etária } \\
\hline 10 a 20 anos & $21(17,4)$ \\
\hline Maior que 20 anos & $100(82,6)$ \\
\hline \multicolumn{2}{|l|}{ Situação conjugal ${ }^{\star}$} \\
\hline Solteira & $104(88,9)$ \\
\hline Casada & $8(6,8)$ \\
\hline Divorciada/viúva & $5(4,3)$ \\
\hline \multicolumn{2}{|l|}{ Raça/Cor } \\
\hline Não negra & $10(8,3)$ \\
\hline Negra & $111(91,7)$ \\
\hline \multicolumn{2}{|l|}{ Escolaridade $^{*}$} \\
\hline Menos de 7 anos de estudo & $85(80,9)$ \\
\hline 7 anos ou mais de estudo & $20(19,0)$ \\
\hline \multicolumn{2}{|l|}{ Tipo da violência prévia** } \\
\hline Física & $100(65,8)$ \\
\hline Sexual & $8(5,3)$ \\
\hline Psicológica & $33(21,7)$ \\
\hline Outras & $11(7,2)$ \\
\hline \multicolumn{2}{|l|}{ Meio de agressão da violência prévia** } \\
\hline Força corporal & $54(42,2)$ \\
\hline Arma de fogo & $20(15,6)$ \\
\hline Objeto contundente/cortante/perfurante & $27(21,1)$ \\
\hline Ameaça & $20(15,6)$ \\
\hline Outros & $7(5,5)$ \\
\hline \multicolumn{2}{|l|}{ Relação agressor/vítima** } \\
\hline Parceiro/Ex-parceiro íntimo & $40(51,9)$ \\
\hline Filho & $3(3,9)$ \\
\hline Irmão & $3(3,9)$ \\
\hline Desconhecido & $9(11,7)$ \\
\hline Outros & $22(28,6)$ \\
\hline \multicolumn{2}{|l|}{ Reincidência da violência } \\
\hline $\operatorname{Sim}$ & $44(36,4)$ \\
\hline Não & $77(63,6)$ \\
\hline \multicolumn{2}{|l|}{ Local de ocorrência do óbito } \\
\hline Hospital/outros serviços de saúde & $50(41,3)$ \\
\hline Domicílio & $23(19,0)$ \\
\hline Via pública & $39(32,2)$ \\
\hline Outros & $9(7,4)$ \\
\hline \multicolumn{2}{|l|}{ Causa do óbito } \\
\hline Disparo de arma de fogo & $54(44,6)$ \\
\hline Objeto cortante ou penetrante & $31(25,6)$ \\
\hline Objeto contundente & $15(12,4)$ \\
\hline Enforcamento, estrangulamento e sufocação & $3(2,5)$ \\
\hline Agressão por outros meios & $18(14,9)$ \\
\hline
\end{tabular}

*Número/porcentagem de ignorados: Situação conjugal 4/3,3\%; Escolaridade 16/13,2\%; * *Uma notificaçãa de violência pode ter mais de um tipo de violência, mais de um meio de agressão e mais de um agressor

as mulheres expostas à violência tiveram um risco estimado de mortalidade 8,3 vezes maior que o da população geral de mulheres e cerca de 100 mulheres morreram, semanalmente, como consequência direta ou indireta da exposição à violência. (17) Outra pesquisa revelou que o risco de morte foi de, no mínimo, 42 vezes maior para mulheres com registros de violência, em comparação com a po- pulação feminina em geral. ${ }^{(16)}$ As mulheres adultas, com notificação de violência física, possuíam um risco 112,2 vezes maior para a morte por agressáo do que a população geral. ${ }^{(16)}$

Foram identificados 121 homicídios que tinham notificações prévias de violência, dos quais 20 estavam registrados no Sistema de Informaçôes sobre Mortalidade como causas externas de intenção indeterminada. Esses resultados podem demonstrar subnotificação de homicídios de mulheres. Verificou-se que quase $9 \%$ das vítimas de homicídios tinham notificação prévia de violência no Sistema de Informação de Agravos de Notificação.

É provável que a proporção de mulheres vítimas de violência esteja subnotificada e os dados do Sistema de Informação de Agravos de Notificação não permitam retratar isoladamente a violência contra a mulher, porém fornecem indicativos importantes para as açóes de prevenção. $\mathrm{O}$ panorama $\mathrm{da}$ violência contra as mulheres no Brasil destaca, entre os seus resultados, a subnotificação no registro dos principais indicadores, com intensidade variada entre as Unidades da Federação. ${ }^{(18)}$ Muitas vezes, as mulheres vítimas de violência silenciam sobre o fato e os principais fatores para isso podem ser de natureza microssistêmicos (autoculpa, preocupação com a família ou com os filhos) ou macrossistêmicos (expectativas da sociedade, normalização da violência e valores religiosos).

O Sistema de Informação de Agravos de Notificação continha 32.308 notificaçóes de violência contra mulher, em todo período estudado. Somente no ano de 2016, de acordo com indicadores criminais da Polícia Civil de Pernambuco, houve 31.081 registros de violência doméstica e intrafamiliar contra mulheres. ${ }^{(19)}$ Reconhecidamente, o número de casos de violência é superior aos notificados pela saúde, pois nem todos os tipos de violência demandam assistência nos serviços de saúde ou, quando há atendimento de saúde, não são notificados, entretanto podem gerar denúncias às autoridades policiais, o que pode justificar a existência de um maior quantitativo de casos nos registros policiais. ${ }^{(5,20)}$

As mulheres vítimas de violência, atendidas nos serviços de saúde, caracterizam uma oportunidade para a prevenção de homicídios por meio da integração da 
avaliação de risco (identificar casos de alto risco) e reforçam a necessidade de intervençóes de justiça penal e de serviço social. ${ }^{(16)}$ Embora a triagem para a violência por parceiro, em ambientes de assistência à saúde, seja recomendada por organizaçóes médicas e de enfermagem, ela é de baixo desempenho. Nesse sentido, o treinamento para rastrear as vítimas de violência nos atendimentos nas emergências pode contribuir para identificação dessas vítimas, realização de intervençôes e aumento nos encaminhamentos necessários. ${ }^{(21)}$

Nos Estados Unidos, 11,2\% das mulheres vítimas de homicídio sofreram algum tipo de violência no mês anterior a sua morte. ${ }^{(22)} \mathrm{Na}$ Itália, $52,4 \%$ das mulheres vítimas de homicídios tinham registros de admissão em serviços de emergência, por agressóes físicas, nos 24 meses anteriores a sua morte. ${ }^{(23)}$ No Brasil, de 2011 a 2015, houve 23.278 óbitos de mulheres por homicídio e, dentre essas, 2.559 (10,9\%) tinham notificação prévia de violência. ${ }^{(16)}$

As características das vítimas reforçam a discussão de que a raça/cor negra e a baixa escolaridade, relacionadas à situação socioeconômica, interferem na vulnerabilidade a agravos violentos. ${ }^{(24)} \mathrm{Um}$ estudo mostrou que mulheres negras tiveram a maior taxa de morte por homicídio (4,4 por 100.000), quando comparadas a outras raças. ${ }^{(22)}$

As agressóes cometidas pelo parceiro ou ex-parceiro íntimo, e por meio de disparo de arma de fogo, predominaram neste estudo. Um estudo com mulheres atendidas na atenção básica, na Espanha, mostrou que, entre aquelas que relataram já ter sofrido violência, $42,7 \%$ foram agredidas por seu parceiro íntimo. ${ }^{(25)}$ Outros autores mostram que os casos em que o perpetrador tinha histórico de agressóes, a probabilidade das vítimas sofrerem uma lesão fatal na sua residência é 2,4 vezes maior. ${ }^{(26)}$

No Peru, foi identificado que os perpetradores de feminicídios eram, predominantemente, parceiros íntimos, tinham como principais motivos ciúmes e não aceitar separaçóes. ${ }^{(27)}$ Uma pesquisa desenvolvida nos Estados Unidos mostrou que 60,2\% das vítimas de homicídio tinham histórico de violência prévia e que $5,7 \%$ foram agredidas no mês anterior a sua morte. ${ }^{(28)}$

As mortes por meio de arma de fogo foram mais frequentes, concordando com resultados encontra- dos em pesquisas nacionais e internacionais. ${ }^{(28-30)}$ Nos Estados Unidos, no ano de 2015, 64\% dos homicídios de mulheres foram perpetrados por um parceiro íntimo e as armas de fogo figuraram proeminente nessas mortes. Cerca de $60 \%$ dos homens matam usando a arma de fogo. ${ }^{(22,31)}$ Estudos sugerem que a restrição legal à posse de armas de fogo por pessoas com histórico de violência ao parceiro íntimo pode prevenir homicídios de mulheres. ${ }^{(32,33)}$

Os objetos cortantes ou penetrantes constituíram a segunda causa dos homicídios, neste estudo. $\mathrm{O}$ uso de armas cortantes, objetos contundentes ou métodos que usam a força corporal pode representar um meio para agredir a vítima durante um conflito agudo, enquanto que a arma de fogo indica uma clara intenção de assassinar a vítima (premeditação). ${ }^{(34)}$ No Peru, entre 2009 e 2014, observou-se que $40,9 \%$ dos homicídios de mulheres foram, sabidamente, premeditados. ${ }^{(27)}$

Os serviços de saúde são locais em que, muitas vezes, as vítimas de violência buscam assistência. As equipes de saúde podem se deparar com casos de violência, desde a atenção primária, até o nível terciário da atenção à saúde. ${ }^{(21,25)}$ É necessária a compreensão de que os serviços de saúde precisam atuar de forma integrada com os demais setores da rede de proteção da vítima de violência. Dessa forma, a notificação da violência, por meio da ficha de notificação de violência interpessoal/autoprovocada, quando articulada com o encaminhamento da vítima para os demais serviços (segurança pública, assistência social) pode interromper o ciclo de violência. ${ }^{(16)}$ Quanto aos gestores da saúde, entende-se que necessitam conhecer normas e/ou protocolos existentes para o enfrentamento da violência contra a mulher, para, dessa forma, ofertar assistência às vítimas de violência. ${ }^{(35)}$

Este estudo apresenta algumas limitações. Em primeiro lugar, a qualidade do relacionamento é limitada pela ausência de identificadores únicos e pela qualidade dos dados (registros truncados e informaçôes ausentes ou ambíguas). Talvez não tenha sido possível identificar todas as correspondências, perdidas devido aos dados ausentes em alguns registros. Para a melhor utilização dos dados pareados, é importante avaliar a qualidade dos processos de vincula- 
ção e compreender as limitaçóes e o viés que os erros de vinculaçáo podem introduzir nos resultados do estudo. A alta qualidade do relacionamento das bases é fundamental para a robustez dos resultados.

Em segundo lugar, os conjuntos de dados não se sobrepóem exatamente, logo o número esperado de correspondências é desconhecido. Em terceiro lugar, dados que seriam relevantes para uma análise mais detalhada da situação da violência contra a mulher, como por exemplo, os registros de ocorrências policiais, apresentam restriçôes para a disponibilização. Neste estudo, mesmo após pedido oficial, os dados não foram disponibilizados pela Secretaria de Defesa Social. Em que pese essas limitaçóes, o linkage é relevante para o conhecimento do cenário da violência contra mulher e para a elaboraçáo de um conjunto de açóes que visem a sua redução.

\section{Conclusão}

O perfil das mulheres vítimas de violência foi de solteiras, negras e com baixa escolaridade. A agressão física foi o tipo de violência mais notificado, cometida por parceiro/ex-parceiro íntimo. A maior parte dos óbitos ocorreu por arma de fogo. Mulheres com notificação prévia de violência tiveram risco de homicídio superior, quando comparadas com a população geral de mulheres. Reduzir a violência letal contra as mulheres requer medidas abrangentes, abordando fatores individuais, sociais, econômicos, culturais e situacionais. Os resultados deste estudo poderão contribuir com a ampliação dos conhecimentos, na área da saúde pública, sobre violência contra a mulher. A descrição dos homicídios de mulheres, com registros prévios de violência, pode auxiliar no planejamento de açóes da rede intersetorial que atua no enfretamento da violência contra mulheres, bem como para a formulação de políticas públicas de proteção e prevenção da violência contra mulher.

\section{Agradecimentos}

Á Coordenação de Aperfeiçoamento de Pessoal de Nível Superior. CAPES código 001-bolsa de mestrado.

\section{Colaborações}

Barros SC, Pimentel DR, Oliveira CM e Bonfim CV declaram que contribuíram com a concepção do estudo, análise e interpretação dos dados, redação do artigo, revisão crítica relevante do conteúdo intelectual e aprovação da versão final a ser publicada.

\section{Referências}

1. Zaghloul NM, Megahed HM. A descriptive medico-legal study of female deaths in cairo governorate, Egypt. J Forensic Leg Med. 2019;66:2532.

2. Websdale N, Ferraro K, Barger SD. The domestic violence fatality review clearinghouse: introduction to a new National Data System with a focus on firearms. Inj Epidemiol. 2019;6(6):6.

3. Lutgendorf MA. Intimate Partner Violence and Women's Health. Obstet Gynecol. 2019;134(3):470-80.

4. García-Moreno C, Hegarty K, d'Oliveira AF, Koziol-McLain J, Colombini $M$, Feder $G$. The health-systems response to violence against women. Lancet. 2015;385(9977):1567-79.

5. Garcia LP. The invisible magnitude of violence against women. Epidemiol Serv Saude. 2016;25(3):451-4.

6. Brasil. Ministério da Saúde. Doenças e agravos de notificação. violência doméstica, sexual e/ou outras violências. Brasília (DF): DATASUS; 2020 [citado 2020 Mai 27]. Disponível em: https://datasus.saude. gov.br/acesso-a-informacao/doencas-e-agravos-de-notificacao-de2007-em-diante-sinan/

7. Instituto de Pesquisa Econômica Aplicada. Fórum Brasileiro de Segurança Pública. Atlas da violência 2019. Rio de Janeiro: IPEA; 2019 [cited 2020 Mai 27]. Disponível em: https://www.ipea.gov.br/ portal/images/stories/PDFs/relatorio_institucional/190605_atlas_da_ violencia_2019.pdf. Portuguese.

8. Sayers A, Ben-Shlomo Y, Blom AW, Steele F. Probabilistic record linkage. Int J Epidemiol. 2016;45(3):954-64.

9. Paixão ES, Harron K, Andrade K, Teixeira MG, Fiaccone RL, Costa MD, et al. Evaluation of record linkage of two large administrative databases in a middle income country: stillbirths and notifications of dengue during pregnancy in Brazil. BMC Med Inform Decis Mak. 2017;17(1):108.

10. Hagger-Johnson G, Harron K, Goldstein H, Aldridge R, Gillbert R. Probabilistic linkage to enhance deterministic algorithms and reduce data linkage errors in hospital administrative data. J Innov Health Inform. 2017;24(2):891.

11. Cerel J, Singleton MD, Brown MM, Brown SV, Bush HM, Brancado CJ. Emergency Department Visits Prior to Suicide and Homicide: Linking Statewide Surveillance Systems. Crisis. 2016;37(1):5-12.

12. Austin AE, Vladutiu CJ, Jones-Vessey KA, Norwood TS, Proescholdbell SK, Menard MK. Improved Ascertainment of Pregnancy-Associated Suicides and Homicides in North Carolina. Am J Prev Med. 2016;51(5 Suppl 3):S234-40.

13. Souto RM, Porto DL, Pinto IV, Vidotti CC, Barufaldi LA, Freitas MG, et al. Rape and pregnancy of girls aged up to 13 years in Brazil: characteristics and implications in health during gestation, delivery and childbirth. Cien Saude Colet. 2017;22(9):2909-18. 
14. Instituto Brasileiro de Geografia e Estatística (IBGE). Sistema IBGE de Recuperação Automática - SIDRA. Rio de Janeiro: IBGE; 2019.

15. Pernambuco (Estado) Governo do Estado. Cadernos de Informações em Saúde. Recife: 2019 [citado 2019 Set 15]. Disponível em: http://portal. saude.pe.gov.br/aplicativo/secretaria/cadernos-de-informacoes-em-saude

16. Barufaldi LA, Souto RM, Correia RS, Montenegro MM, Pinto IV, Silva $\mathrm{MM}$, et al. Gender violence: a comparison of mortality from aggression against women who have and have not previously reported violence. Cien Saude Colet. 2017;22(9):2929-38.

17. Sandoval GA, Marinho F, Delaney R, Pinto IV, Lima CM, Costa RM, et al. Mortality risk among women exposed to violence in Brazil: a population-based exploratory analysis. Public Health. 2020;179:45-50

18. Brasil. Senado Federal. Panorama da violência contra as mulheres no Brasil. Brasilia (DF): Observatório da Mulher Contra a Violência; 2018 [citado 2020 Mai 27]. Disponível em: https://www.ipea.gov.br/portal/images/stories/PDFs/ relatorio_institucional/190605_atlas_da_violencia_2019.pdf.

19. Pernambuco (Estado) Governo do Estado. Indicadores Criminais de Pernambuco. Violência doméstica e familiar contra a mulher. Recife: Secretaria de Defesa Social de Pernambuco, 2019 [citado 2019 Set 15]. Disponível em: http://www.senado.gov.br/institucional/ datasenado/omv/indicadores/relatorios/BR-2018.pdf

20. Teofilo MM, Kale PL, Eppinghaus AL, Azevedo OP, Farias RS, Maduro Neto JP, et al. Violência contra mulheres em Niterói, Rio de Janeiro: informações do Sistema de Vigilância de Violências e Acidentes (20102014). Cad Saude Colet. 2019;27(4):437-47.

21. Karnitschnig $L$, Bowker S. Intimate partner violence screening in the emergency department: a quality improvement project. J Emerg Nurs. 2020;46(3):345-53.

22. Petrosky E, Blair JM, Betz CJ, Fowler KA, Jack SP, Lyons BH. Racial and ethnic differences in homicides of adult women and the role of intimate partner violence - United States, 2003-2014. MMWR Morb Mortal Wkly Rep. 2017;66(28):741-6.

23. Mamo C, Bianco S, Dalmasso M, Girotto M, Mondo L, Penasso M. Are emergency department admissions in the past two years predictors of femicide? Results from a case-control study in Italy. J Fam Violence. 2015;30(7):853-8.

24. Berezin J, Gale S, Nuru-Jeter A, Lahiff M, Auerswald C, Alter H. violent injury and neighborhood racial/ethnic diversity in Oakland, California. J Urban Health. 2017;94(6):882-91.
25. Martín-Baena D, Montero-Piñar I, Escribà-Agüir V, Vives-Cases C. Violence against young women attending primary care services in Spain: prevalence and health consequences. Fam Pract. 2015;32(4):381-6.

26. Yousuf S, McLone S, Mason M, Snow L, Gall C, Sheehan K. Factors associated with intimate partner homicide in Illinois, 2005-2010: Findings from the Illinois Violent Death Reporting System. J Trauma Acute Care Surg. 2017;83(5S Suppl 2):S217-21.

27. Ayala Quintanilla BP, Taft A, McDonald S, Pollock W. An examination of femicides in Peru between 2009 and 2014. Int J Gynaecol Obstet. 2016;134(3):342-3.

28. Jiang $Y$, DeBare $D$, Shea LM, Viner-Brown S. Violence against women: injuries and deaths in Rhode Island. R I Med J (2013). 2017;100(12):24-8.

29. Souza ER, Meira KC, Ribeiro AP, Santos JD, Guimarães RM, Borges $L F$, et al. Homicides among women in the different Brazilian regions in the last 35 years: an analysis of age-period-birth cohort effects. Cien Saude Colet. 2017;22(9):2949-62.

30. Borges LM, Lodetti MB, Tridapalli AL, Machado GS. [Partner homicide in the greater São Paulo and greater Florianópolis: news published in newspapers]. Rev. Interinst. Psicol. 2016;9(2):227-40. Portuguese.

31. United States Department of Justice; Federal Bureau of Investigation. Uniform Crime Reporting Program Data: Supplementary Homicide Reports, 2015. Ann Arbor, Ml: Inter-university Consortium for Political and Social Research; 2017.

32. Díez C, Kurland RP, Rothman EF, Bair-Merritt M, Fleegler E, Xuan Z, et al. State intimate partner violence-related firearm laws and intimate partner homicide rates in the United States, 1991 to 2015. Ann Intern Med. 2017;167(8):536-43.

33. McPhedran S. An Evaluation of the impacts of changing firearms legislation on australian female firearm homicide victimization rates. Violence Against Women. 2018;24(7):798-815.

34. Carmichael H, Jamison E, Bol KA, Mclntyre R Jr, Velopulos CG. Premeditated versus "passionate": patterns of homicide related to intimate partner violence. J Surg Res. 2018;230:87-93.

35. Batista KB, Schraiber LB, D'Oliveira AF. Gestores de saúde e 0 enfrentamento da violência de gênero contra as mulheres: as políticas públicas e sua implementação em São Paulo, Brasil. Cad Saude Publica. 2018;34(8):e00140017. 